\title{
New Lead Free Halide Double Perovskite Materials: Potential Substitutes Towards Green Technology and Stable Optoelectronic Application
}

Muskan Nabi ( $\square$ muskannabi7500@gmail.com )

Jiwaji University

Dinesh C. Gupta

Jiwaji University

\section{Research Article}

Keywords: halide double perovskites, absorption coefficient, structural stability, optoelectronic materials, semiconductors.

Posted Date: March 4th, 2021

DOI: https://doi.org/10.21203/rs.3.rs-263059/v1

License: (c) (i) This work is licensed under a Creative Commons Attribution 4.0 International License.

Read Full License 
New lead-free halide double perovskite materials: potential substitutes towards green technology and stable optoelectronic application

\author{
Muskan Nabi* and Dinesh C. Gupta ${ }^{\#}$ \\ Condensed Matter Theory Group, School of Studies in Physics, \\ Jiwaji University, Gwalior - 474011 (INDIA) \\ e-mail: sosfizix@gmail.com ${ }^{\#}$,muskannabi7500@gmail.com*
}

\begin{abstract}
:
Explorations of new stable lead free perovskites have currently achieved substantial interest in the field of photovoltaics and optoelectronics as it tries to overcome the instability issue and avoid toxicity related with the lead based perovskites. We herein not only comprehensively tried to explain the experimentally synthesized two inorganic halide double perovskite materials but exploring their broader structural stability and also provide us a guideline to better understand their possible potential applications. For this purpose we performed density functional theory to investigate the structural, electronic, optical, elastic and thermoelectric properties of these materials. The stability of these cubic materials is validated by optimizing the structure, from the tolerance factor, mechanical stability test. These materials are found to be small band gap semiconductors with outshining optoelectronic performance. Due to high optical absorption, high conductivity and low reflectivity they have great potential to be used as a light absorbing material for photovoltaic application. Because, of small band gap we also tried to explore the variation of various transport properties with chemical potential. The semiconducting nature of materials results in ZT close to unity predicting its excellent application in thermoelectric technology.
\end{abstract}

\title{
Introduction:
}

Due to increase in energy demands there has been an increase in consumption of fossil fuels which sufficiently contributes to the pollution levels leading to global warming. So, an ecofriendly power source is needed to meet the energy crises. In order to overcome this issue, solar cells made up of silicon proved to be an ideal solution. However, besides being costlier the silicon based solar cells have far less power conversion efficiency also with complicated production procedure. So, there is a sustained research interest toward alternative photovoltaic materials produced with cost-competitive, facile, and environmentally friendly technologies. In 
this field perovskite solar cells have gained much progress during last few decades increasing the efficiency from $3.8 \%$ in 2009 to $22.7 \%$ in 2017 at the lab scale [1-5]. Hybrid halide perovskites with a general formula $\mathrm{ABX}_{3}$ where $\mathrm{A}$ is monovalent cation such as methylammonium $\left(\mathrm{MA}^{+}\right)$, formamidium $\left(\mathrm{FA}^{+}\right)$and $\mathrm{B}$ is divalent cation such as $\mathrm{Pb}^{2+}$ and $\mathrm{X}$ are halides such as $\mathrm{Cl}, \mathrm{Br}, \mathrm{I}$ are the most commonly studied materials for optoelectronic applications. But there are two main issue associated with their commercialization such as instability and toxicity [6-7]. Due to less stability of organic cations such as methylammonium $\left(\mathrm{MA}^{+}\right)$, formamidium $\left(\mathrm{FA}^{+}\right)$against the environment condition cesium halide perovskites proved to be saviors due to their thermodynamic and environmental stability. Since lead is a very toxic heavy element has hazardous effects not only to environment but also on human beings. Therefore, it is very important to reduce or eliminate lead from photovoltaic devices [8-9]. So, keeping this in view research is going on to replace those lead based perovskites with other non-toxic elements. The replacement for lead must fulfill certain criteria in order to match the excellent performance of these lead based perovskites. The alternative to lead must be low-cost, easily recycled, should exhibit excellent optoelectronic properties. In addition to be competitive with current established photo voltaic (PV) technologies they should satisfy some commercial necessities like flexibility, long-term stability and scalability [10-11]. The issue is being addressed by the replacement of lead-based perovskites by environmental friendly lead free halide based perovskites. The success arises because of these semi-conducting halide perovskites possess outstanding optoelectronic properties such as high optical absorption coefficient, band gap that can be tuned, long carrier recombination lifetimes, high carrier mobility, small electron/hole effective masses and high molar extinction coefficient.

A chase is being launched to explore halide double perovskites materials, among which Bi-based family have drawn a remarkable interest. $\mathrm{Cs}_{2} \mathrm{MBiCl}_{6}(\mathrm{M}=\mathrm{Ag}, \mathrm{Cu}, \mathrm{Na})$ and $\mathrm{Cs}_{2} \mathrm{MBiCl}_{6}(\mathrm{M}=\mathrm{K}, \mathrm{Rb}$ and Cs) have shown excellent stability and good optoelectronic application with smaller and larger band gaps correspondingly [12]. Theoretically, $\mathrm{Cs}_{2} \mathrm{NaBX}_{6}(\mathrm{~B}=\mathrm{Sb}, \mathrm{Bi} ; \mathrm{X}=\mathrm{Cl}, \mathrm{Br}, \mathrm{I})$ have been predicted, among which $\mathrm{Cs}_{2} \mathrm{NaBiCl}_{6}$ have shown larger band gaps [13]. A lot of literature is available to justify their application in thermoelectricity and optoelectronic application [14-21]. In this paper we have tried to explore the experimentally synthesized small band gap halide double perovskites $\mathrm{Cs}_{2} \mathrm{CuMCl}_{6}(\mathrm{M}=\mathrm{Sb}, \mathrm{Bi})$ [22-30]. The nanocrystals of these halide doubled perovskites and other related materials have been experimentally reported. Besides, the 
experimental synthesis, these novel materials have also been studied through density functional simulations. DFT simulations have been verified to be one of the most versatile, accurate and efficient methods to explore and find out the new materials with fascinating properties. Motivated by their small band gap and unmatchable desirable properties, we have tried to explore these materials for optoelectronic application and extended our study to unravel their thermoelectric properties which they equally justify. Our main goal is to explore these materials for superior applications. It is universally acknowledged that significant effort is needed to discover more capable materials and modulate the properties for their further applications.

Keywords: halide double perovskites, absorption coefficient, structural stability, optoelectronic materials, semiconductors.

\section{Computational Methods:}

The first principle method with the help of Wein $2 k$ simulation code [31] is used to calculate the electronic structure, optical and transport properties of the materials. The ground state properties are calculated by evaluating the only two unknown terms (exchange and correlation) in the Kohn- Sham equation properly. For the said purpose, different approximation methods like generalized gradient approximation (GGA), onsite coulomb interaction (GGA+U) and modified Becke -Johson (mBJ) were utilized [32-33]. The unit cell volume is divided into muffin tin spheres where wave function shows atomic like character and interstitial space which exhibit Bloch plane wave character. So, in order to ensure that there is less charge in interstitial region and to avoid the charge leakage $R_{M T} K_{\max }=7$ and $l_{\max }=10$ is chosen where $R_{M T}$ is smallest muffin tin radii and $\mathrm{K}_{\max }$ represent maximum value of $\mathrm{k}$. To obtain the convergence of results the unit cell in the k-space is divided into a dense mesh of 1000-k points for integration over the Brillouin zone. As the thermoelectric parameters are sensitive to $\mathrm{k}$ point sampling therefore a high dense of $150,000 \mathrm{k}$ points is utilized to calculate the same. The iterations for charge convergence between successive cycles converge up to 0.0001 e and energy up to 0.0001 Ry to obtain better results. The thermoelectric properties are determined under the approximation of constant relaxation time $(\tau)$ with the help of BoltzTraP code [34]. With the help of Gibbs2 code [35] we tried to evaluate some of the thermodynamic parameters like Debye temperature and Grüneisen parameter. 


\section{Structural properties:}

The structural stability of the materials can be determined by various factors like by utilizing the Birch Murnaghan equation of state [36], Goldschmidt's rule [37] and from the effective ionic radii or bond length. In addition to structural stability the band structure and carrier transport performance of materials and their specific application to a large extent is predominately determined by the combination of cations and anions. Because of less number of experimentally synthesized halide double perovskites their theoretical determined structure proves to be an ideal solution. The band structure of halide double perovskites with general formula $\mathrm{A}_{2} \mathrm{~B}(\mathrm{I}) \mathrm{B}^{\prime}(\mathrm{III}) \mathrm{X}_{6}$ are predominantly decided by B (I)-, B(III)- (or B(IV)-) and X-site atoms. Fig. S1 (a) (supplementary information) shows a possible combination of different cations in the periodic table for the possible formation of halide double perovskites [38]. The correct combination of these cations leads to the excellent properties of these materials. In the present case the structural optimization of the titled halide double perovskites is determined by utilizing the BirchMurnaghan equation of state which justifies the cubic stability with non-magnetic ground state as the stable state as shown in Fig. 1 (a, b). Also, the cubic stability is determined from tolerance factor. All the stabilized parameters of these materials are quoted in Table 1 which agrees well with the previous reported results [22-23]. The pictorial representation of the titled perovskite in which cesium is enclosed by a cage of 12 halide atoms $(\mathrm{Cl})$ while as $d$ block element $\mathrm{Cu}$ and $p$ group elements $(\mathrm{Sb} / \mathrm{Bi})$ lie in octahedral of halide atoms having coordination 6 to these atoms is shown in Fig. S1 (b) (supplementary information).

Table 1: The optimized lattice parameters of Cubic $\mathrm{Cs}_{2} \mathrm{CuMCl}_{6}(\mathrm{M}=\mathrm{Sb}$, Bi) with space group $F m 3 m$ in both spin polarized and non-polarized states.

\begin{tabular}{|c|c|c|c|c|c|c|c|}
\hline Compound & State & $\mathbf{a}(\AA)$ & $\begin{array}{l}\text { Previous } \\
\text { Reported }\end{array}$ & $\mathbf{V}(\stackrel{\AA}{\mathbf{A}})^{\mathbf{3}}$ & $B(\mathbf{G P a})$ & $\mathbf{B}^{\prime}$ & $E_{0}(e V)$ \\
\hline \multirow[t]{2}{*}{$\mathrm{Cs}_{2} \mathrm{CuSbCl}_{6}$} & FM & 10.53 & \multirow{2}{*}{$\begin{array}{l}10.52^{[22]} \\
10.52^{[23]}\end{array}$} & 1045.31 & 32.70 & 5.06 & -720492.60 \\
\hline & NM & 10.53 & & 1045.33 & 32.67 & 5.02 & -720492.80 \\
\hline \multirow[t]{2}{*}{$\mathrm{Cs}_{2} \mathrm{CuBiCl}_{6}$} & FM & 10.64 & \multirow{2}{*}{$\begin{array}{c}10.61^{[22]} \\
10.33^{[24]}\end{array}$} & 1079.05 & 31.72 & 4.84 & -1131156.69 \\
\hline & NM & 10.64 & & 1078.93 & 31.62 & 5.09 & -1131156.70 \\
\hline
\end{tabular}


Figure $1(\mathrm{a}, \mathrm{b})$ : Energy versus volume optimization curve of $\mathrm{Cs}_{2} \mathrm{CuMCl}_{6}(\mathrm{M}=\mathrm{Sb}, \mathrm{Bi})$ in both spin polarized and nonpolarized states.
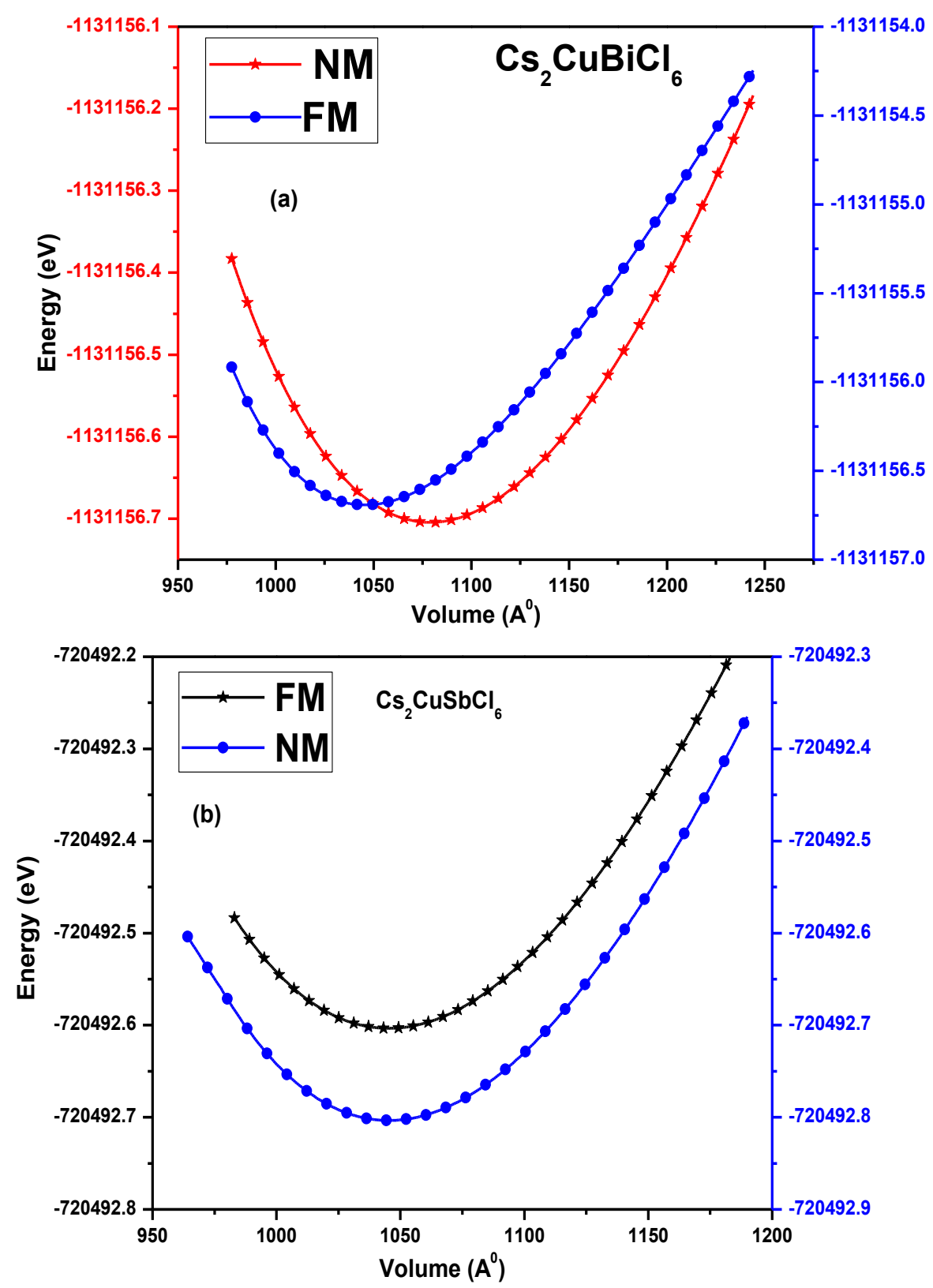

\section{Electronic properties:}

The application of any materials is profoundly characterized by the electronic properties which include band structure and distribution of electrons in these bands [39-41]. Herein, with the assistance of density functional theory, we have evaluated the electronic properties of the considered theoretically inorganic halide double perovskites (supplementary information). The 
band structure calculated via non-spin polarization through different approximation methods is given in Fig. S2 (a, b) and it is clear that the Fermi level is unoccupied indicating their semiconducting nature. Also, the valence band maxima and conduction band minima are located at different points in k-space resulting in indirect band gap. The band gap calculated for $\mathrm{Cs}_{2} \mathrm{CuSbCl}_{6}$ and $\mathrm{Cs}_{2} \mathrm{CuBiCl}_{6}$ by GGA and $\mathrm{GGA}+\mathrm{U}$ is found to be $\sim 0.61 \mathrm{eV}$ and $\sim 0.89 \mathrm{eV}$ respectively. While using $\mathrm{mBJ}$ potential we observe they have indirect band gaps of $\sim 1.80 \mathrm{eV}$ and $\sim 1.00 \mathrm{eV}$ which are closer to experimental values. With the application of $\mathrm{mBJ}$ the energy band mostly populated of $\mathrm{Cl}-\mathrm{p}$ and $\mathrm{Cu}-d$ stated are pushed away from Fermi level resulting in a wider band gap given in Table 2 .

The qualitative description of the valence band and conduction band and the energy states associated with them is illustrated with the help of the density of states as shown in Fig. 2 (a, b, c). In $\mathrm{Cs}_{2} \mathrm{CuMCl}_{6}(\mathrm{M}=\mathrm{Sb}, \mathrm{Bi})$ the distribution of energy states is as $\mathrm{Cu}-d$ and $\mathrm{Cl}-p$ are completely filled below the Fermi level and lie in valence band while as $\mathrm{M}-p(\mathrm{M}=\mathrm{Sb}, \mathrm{Bi})$ and $\mathrm{Cl}-p$ lie empty in the conduction band hybridizing with each other resulting in a small band at Fermi level. The oxidation state of the constituent atoms and the number of remaining valence electrons in their respective oxidation states are the factors that determine whether energy states would be in the valence band or conduction band or occupy the Fermi level. The oxidation state of $\mathrm{Cs}$ and $\mathrm{Cu}$ is +1 state, $\mathrm{Cl}$ has -1 oxidation state while the main block elements $\mathrm{Sb}$ and $\mathrm{Bi}$ are in +3 oxidation state. Therefore, in $\mathrm{Cs}_{2}^{+1} \mathrm{Cu}^{+1} \mathrm{M}^{+3} \mathrm{Cl}_{6}^{-1}(\mathrm{M}=\mathrm{Sb}, \mathrm{Bi})$ oxidation state the charge is balanced; moreover, the constituents have filled the core with only paired electrons. Among all the states, most interested states are $p$-states of $\mathrm{Cl}$ and $d$-states of $\mathrm{Cu}$ which are in the vicinity of Fermi level. The $p$-states of $\mathrm{Cl}$ gets electrons from cations are filled and happen to be in VB. The $\mathrm{d}$ states in the octahedral field mostly split into triplet $d$ - $t 2 g$ and doublet $d$-eg states, $d$ - $t 2 g$ states being at lower energy compared to doublet $d$-eg states. The $d$ - $t 2 g$ can intake a maximum of six electrons ( $3 \uparrow$ and $3 \downarrow$ ) while as deg state is filled by 4-electrons $(2 \uparrow$ and $2 \downarrow)$. Therefore, the electron filling in d-orbitals of $\mathrm{Cu}^{1+}$ is $3 t-2 g(\uparrow), 3 \mathrm{t} 2 \mathrm{~g}(\downarrow), 2 t-e g(\uparrow)$, and 2-eg $(\downarrow)$. All the dorbitals are filled for both spin channels therefore form VB. Moreover, the crystal field energy for the configuration is zero. The constituent atoms have no unpaired electrons resulting in the non-magnetic character of these materials which is also confirmed from the structural optimization. The obtained semi-conducting nature along with small band gap values signifies that they can outshine in optoelectronic applications. 
Table 2: Calculated and reported band gap and nature of band gap in $\mathrm{Cs}_{2} \mathrm{CuMCl}_{6}(\mathrm{M}=\mathrm{Sb}, \mathrm{Bi})$.

\begin{tabular}{|c|c|c|c|c|c|}
\hline Materials & GGA & GGA+U & mBJ & Band gap nature & $\begin{array}{c}\text { Reported } \\
\text { values }\end{array}$ \\
\hline $\mathbf{C s}_{\mathbf{2}} \mathbf{C u S b C l} 6$ & 0.61 & 0.61 & 1.00 & Indirect & $1.66^{[22],}$ \\
\hline $\mathbf{C s}_{\mathbf{2}} \mathbf{C u B i C l}_{\mathbf{6}}$ & 0.89 & 0.89 & 1.20 & Indirect & $\begin{array}{l}0.83^{[23]}, 2.00 \\
\end{array}$ \\
\hline
\end{tabular}

Fig 2 (a): Density of states for $\mathrm{Cs}_{2} \mathrm{CuMCl}_{6}(\mathrm{M}=\mathrm{Sb}, \mathrm{Bi})$ calculated by GGA, GGA+U and $\mathrm{mBJ}$ scheme.
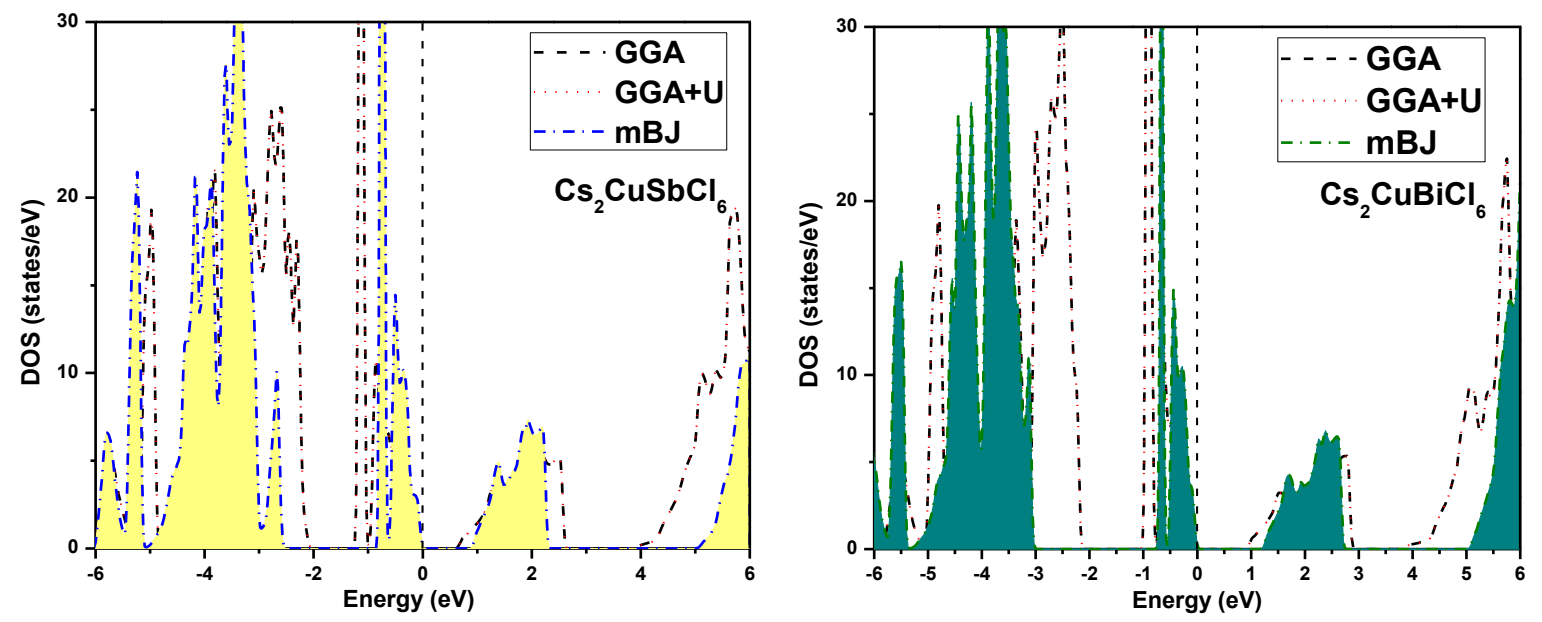

Fig 2 (b): Partial density of states pDOS for $\mathrm{Cs}_{2} \mathrm{CuMCl}_{6}(\mathrm{M}=\mathrm{Sb}, \mathrm{Bi})$ calculated by $\mathrm{mBJ}$ scheme.
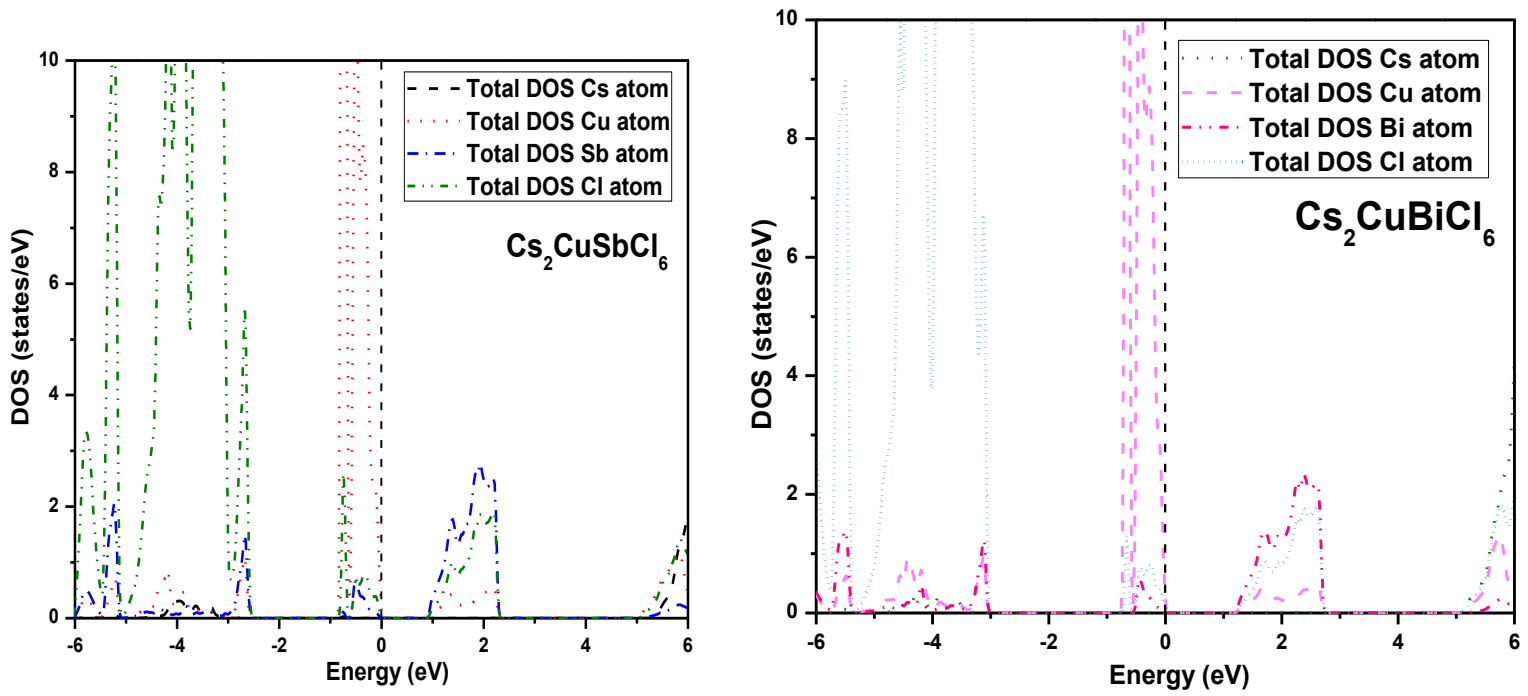
Fig 2 (c): Partial density of states pDOS for $\mathrm{Cs}_{2} \mathrm{CuMCl}_{6}(\mathrm{M}=\mathrm{Sb}, \mathrm{Bi})$ calculated by $\mathrm{mBJ}$ scheme.
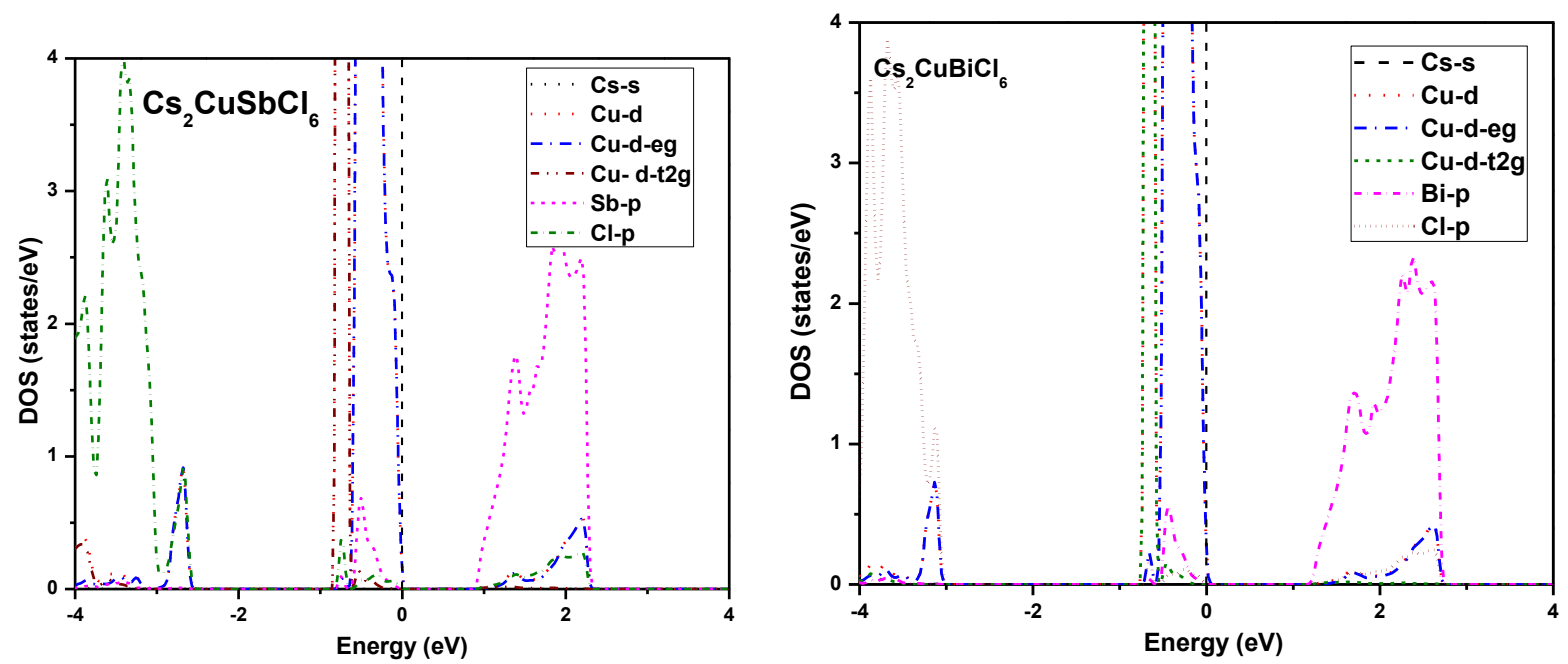

\section{Second-order elastic constants and Mechanical Stability}

The elastic constants and thereby mechanical behavior of these considered double halide perovskites are predicted with the help of the Cubic Elastic package [42]. Equilibrium cubic structure is deformed by applying small strains to predict second order elastic constants. The cubic structure would be mechanically stable only if the deformed structures are at higher energy compared to the cubic phase. This leads to a limiting condition $C_{11}-C_{12}>0, C_{11}>0, C_{44}>$ $0, C_{11}+2 C_{12}>0, C_{12}<B<C_{11}$ [43] for elastic constants to be followed.

The elastic constants help to predict the response of any material to applied stresses. The secondorder elastic constants (SOECs) in the present work have been evaluated utilizing the energystrain approach in the framework of GGA-PBE given in Table 3. All three elastic constants are non-negative and follow the Born's stability criteria [44]. Thereby, advocate the mechanical stability of the materials. From the SOECs, the universal anisotropic factor $\left(A^{U}\right)[45]$ is deduced. The deviation of $\mathrm{A}^{\mathrm{U}}$ from unity signifies titled halide double perovskites are highly anisotropic. The anisotropy mainly originates because of the large difference in longitudinal and shear elastic constants. Using SOEC we have estimated mechanical parameters like Young's (Y), shear (G), and bulk moduli (B), Poisson's ratio $(\sigma)$ using the mathematical relations reported elsewhere [46]. Further, by employing Elate: Elastic tensor analyzer [47], we have analyzed the angular dependence of elastic modulus. The results reflect that Young's modulus and shear modulus are highly anisotropic while bulk modulus is isotropic as happens in cubic crystals as shown in Fig. 
S3 (a,b). In addition to 3D graphical representation of directional elastic properties a quantitative analysis by reporting the minimal and maximal values of each modulus is reported in Table S1 (a). Moreover, by executing the Reuss-Vogoit-Hill scheme [48] we have defined the average values of different elastic moduli. The obtained results are summarized in Table $\mathbf{S 1}$ (b). The $\mathrm{C}_{11-}$ values for both double perovskites are greater than the other two shear elastic constants $\left(\mathrm{C}_{12}\right.$ and $\mathrm{C}_{44}$ ) and also $\mathrm{B}$ being greater than $\mathrm{G}$ reflects that these materials show more resistance for volumetric deformation compared to the shape deformation. The Pugh's ratio (B/G), Poisson's ratio $(\sigma)$, and Cauchy pressure $\left(\mathrm{C}_{P}=\mathrm{C}_{12}-\mathrm{C}_{44}\right)$ [49-51] are greater than their index values of 1.75 , 0.26 , and 0 , respectively as can be seen from Table 3. These values thus signify $\mathrm{Cs}_{2} \mathrm{CuSbCl}_{6}$ and $\mathrm{Cs}_{2} \mathrm{CuBiCl}_{6}$ double perovskites are ductile. So, these materials can be used to design tools of varying shapes.

Additionally, we have simplified the ultrasonic wave velocities of the titled double perovskites using SOECs and the density of the materials [52]. In cubic structure pure longitudinal $\left(V_{L}\right)$ and two transverse $\left(V_{T_{1}}\right.$ and $\left.V_{T_{2}}\right)$ modes only happen along [100], [110] and [111] direction. The magnitude of the sound wave velocity is obtained through the following equation $V=\sqrt{\frac{C_{e f f}}{\rho}}$, [53] where the $C_{\text {eff }}$ for different modes along different directions are defined in Table S1 (c). These wave velocities in turn are used to estimate the average Debye velocity (mean sound velocity $\left.V_{D}\right)$ using relation $V_{D}=\left\{\frac{1}{3}\left(\frac{1}{V_{L}}+\frac{1}{V_{T_{1}}}+\frac{1}{V_{T_{2}}}\right)\right\}^{-\frac{1}{3}}$ [54]. The calculated values of Debye velocity or mean sound velocity are presented in Table 3. Moreover, we have tallied the Debye temperature $\left(\theta_{\mathrm{D}}\right)$ of the $\mathrm{Cs}_{2} \mathrm{CuSbCl}_{6}$ and $\mathrm{Cs}_{2} \mathrm{CuBiCl}_{6}$ perovskites by using the Debye average velocity $V_{D}$ in with equation, $\theta D=\frac{h}{k}\left\{\frac{3 n}{4 \pi} \frac{N_{A} \rho}{M}\right\}^{\frac{1}{2}} V_{D}[55]$. The obtained values of ultrasonic sound velocities and Debye temperature furthermore authenticate the anisotropic nature of the materials. Moreover, the high value of Debye temperature signifies these materials are stable at extreme temperatures and could be used for the fabrication of the devices.

Table 3: Second-order elastic constants (SOECs) obtained by utilizing the energy-strain approach in the framework of GGA-PBE for $\mathrm{Cs}_{2} \mathrm{CuMCl}_{6}(\mathrm{M}=\mathrm{Sb}, \mathrm{Bi})$.

\begin{tabular}{|c|c|c|c|}
\hline \multicolumn{2}{|c|}{ Parameters } & Cs2 $_{2} \mathbf{C u S b C l}$ 6 & $\mathbf{C s}_{2} \mathbf{C u B i C l}_{\mathbf{6}}$ \\
\hline Elastic constants & $\mathrm{C}_{11}(\mathrm{GPa})$ & 57.21 & 56.27 \\
\hline
\end{tabular}




\begin{tabular}{|c|c|c|}
\hline $\mathrm{C}_{12}(\mathrm{GPa})$ & 19.56 & 19.23 \\
\hline $\mathrm{C}_{44}(\mathrm{GPa})$ & 5.05 & 4.05 \\
\hline Bulk modulus (B in GPa) & 32.11 & 31.57 \\
\hline Shear modulus ( $\mathrm{G}$ in $\mathrm{GPa}$ ) & 12.36 & 7.86 \\
\hline Pugh's ratio $(\mathrm{B} / \mathrm{G})$ & 2.59 & 4.01 \\
\hline Young's modulus & 32.86 & 21.77 \\
\hline Poisson's ratio $(\sigma)$ & 0.32 & 0.38 \\
\hline Zener Anisotropy factor $\left(\mathrm{A}^{\mathrm{U}}\right)$ & 0.26 & 0.21 \\
\hline Cauchy pressure & 14.51 & 15.18 \\
\hline Compression velocity $\left(\mathrm{V}_{1}\right.$ in $\left.\mathrm{m} / \mathrm{s}\right)$ & 3410.00 & 3180.00 \\
\hline Shear Sound velocity (Vs in $\mathrm{m} / \mathrm{s}$ ) & 1520.00 & 1370.00 \\
\hline Mean sound velocity $\left(\mathrm{V}_{\mathrm{D}}\right.$ in $\left.\mathrm{m} / \mathrm{s}\right)$ & 3331.02 & 2971.98 \\
\hline Debye temperature $\left(\theta_{\mathrm{D}}\right.$ in $\left.\mathrm{K}\right)$ & 151.17 & 133.38 \\
\hline
\end{tabular}

\section{Optical Properties:}

The optical properties of a material are directly linked to the dielectric function of the material. These properties are being determined by investigating their visible light energy harvest. This is normally done by calculating the bandgap and the absorption coefficients [56]. Ideally, direct low band gap semiconductor materials possess promising optoelectronic applications like photoabsorbers for solar-cell [57]. The optical properties of a material depends on frequency and they are interconnected with each other if we are capable of calculating one e.g., dielectric function we can extort all other properties easily. The optical properties such as the absorption coefficient, refractive index $\mathrm{n}(\omega)$, reflectivity $\mathrm{R}(\omega)$ and conductivity function $\sigma(\omega)$ are obtained from the expression of the real part $\varepsilon(\omega)$ of the dielectric function [58].

First we started with plotting the optical absorption coefficient with photon energy which gives information on the light harvesting capacity of the material. Since, we know that band gap depends inversely upon absorption threshold so materials with higher band gap have narrow absorption in the visible region of electromagnetic spectrum. But the studied materials have smaller band gap showing higher absorption in the visible range as shown in Fig. 3(a). These materials show high absorption coefficient ranging from infrared to ultraviolet region and contains entire visible wavelength range. As the photon energy increases the absorption spectrum increases gradually and highest peak occurs at $6.5 \mathrm{eV}$ which corresponds to maximum 
absorption. This spectrum appeared as a result of electrons exciting from valence to conduction band. The first peak in absorption spectrum for $\mathrm{Cs}_{2} \mathrm{CuSbCl}_{6}\left(\mathrm{Cs}_{2} \mathrm{CuBiCl}_{6}\right)$ is about $2 \mathrm{eV}(2.5 \mathrm{eV})$ which arises due to transitions from $\mathrm{Cu}-d$ to $\mathrm{M}-p(\mathrm{M}=\mathrm{Sb}, \mathrm{Bi})$ while as second peak occurs at 6 $\mathrm{eV}(6.5 \mathrm{eV})$ corresponds to transitions from $\mathrm{Cu}-d$ to $\mathrm{Cl}-p$. The late absorption onset was attributed to the indirect band gap. Further, we tried to investigate the optical conductivity of the materials and plotted in Fig. 3(b). It follows a similar trend as the absorption spectra as shown in Fig. 3(a). Over the entire photo energy range $(0-14 \mathrm{eV})$ conductivity displays high and low peaks with the presence of hump at particular energies. The maximum conductivity is shown at higher energy range. These calculated curves reveal the same trend or same features as observed in case of reported cases [22-23]. The almost similar nature of band structure leads to similar structure of the optical spectra originates from the top of valence band to the bottom of conduction band.

An important physical property in optics which provides information about behavior of light inside a material is refractive index (n). When light is passed through different media, its velocity changes resulting in the variation of refractive of a material. Other important physical quantity which is connected to the light absorption capacity of a material at a particular frequency is called as extinction coefficient. The extinction coefficient is basically complex part of refractive index and represent how electromagnetic wave can propagate in any medium. The variation of refractive index (n) with photon energy is illustrated in Fig. 3(c). The static values of refractive index are 2.3 and 2.0 for $\mathrm{Cs}_{2} \mathrm{CuSbCl}_{6}$ and $\mathrm{Cs}_{2} \mathrm{CuBiCl}_{6}$ respectively. They correspond to the values that can be derived from real part of dielectric function. These obtain a maximum value at around $2 \mathrm{eV}$. From Fig. 3(d) we see that the extinction coefficient can be divided into three main absorption peaks which are centered on different photon energy range. These various peaks arise because of electronic transitions from one level to another. These all together properties coveys that these inorganic halide double perovskites would be a potential lead free alternative in solar cell.

Fig $3(\mathbf{a}, \mathbf{b})$ : variation of optical absorption and conductivity with photon Energy. 

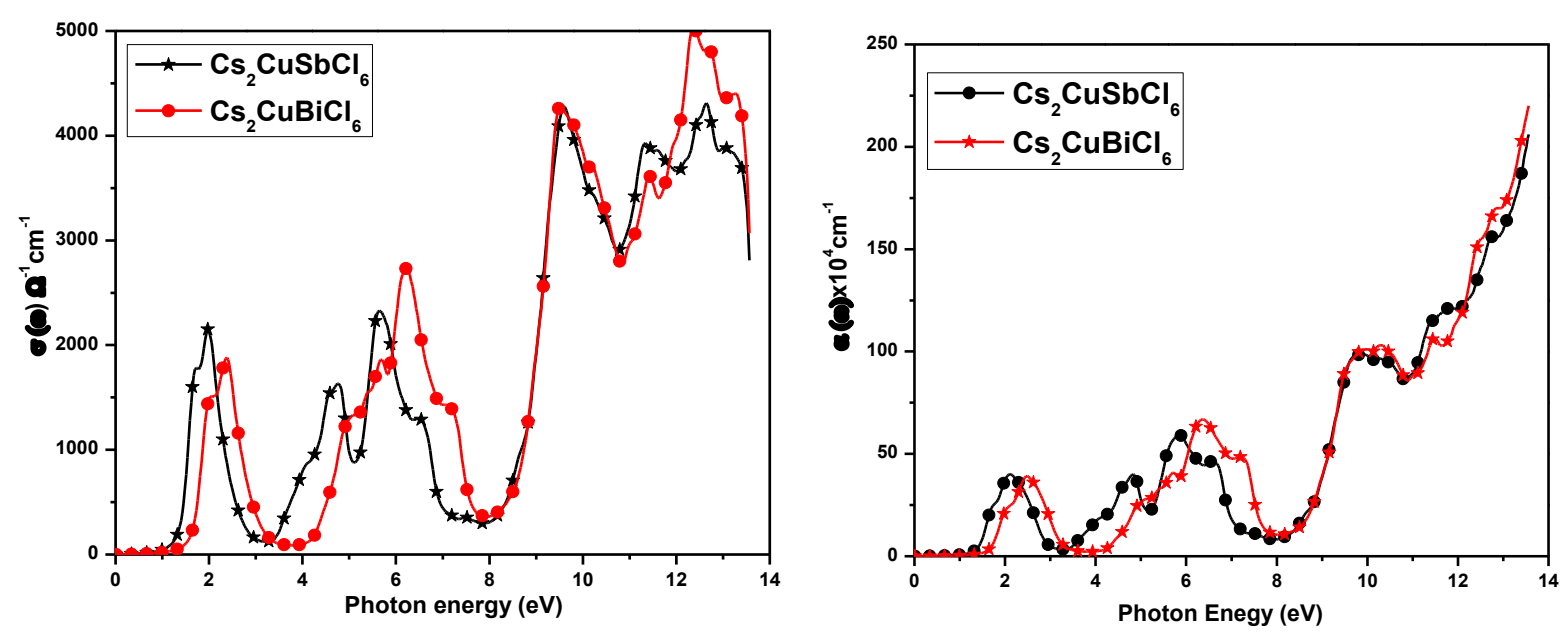

Fig $3(\mathbf{c}, \mathbf{d})$ : variation of refractive index and extinction coefficient with photon Energy.
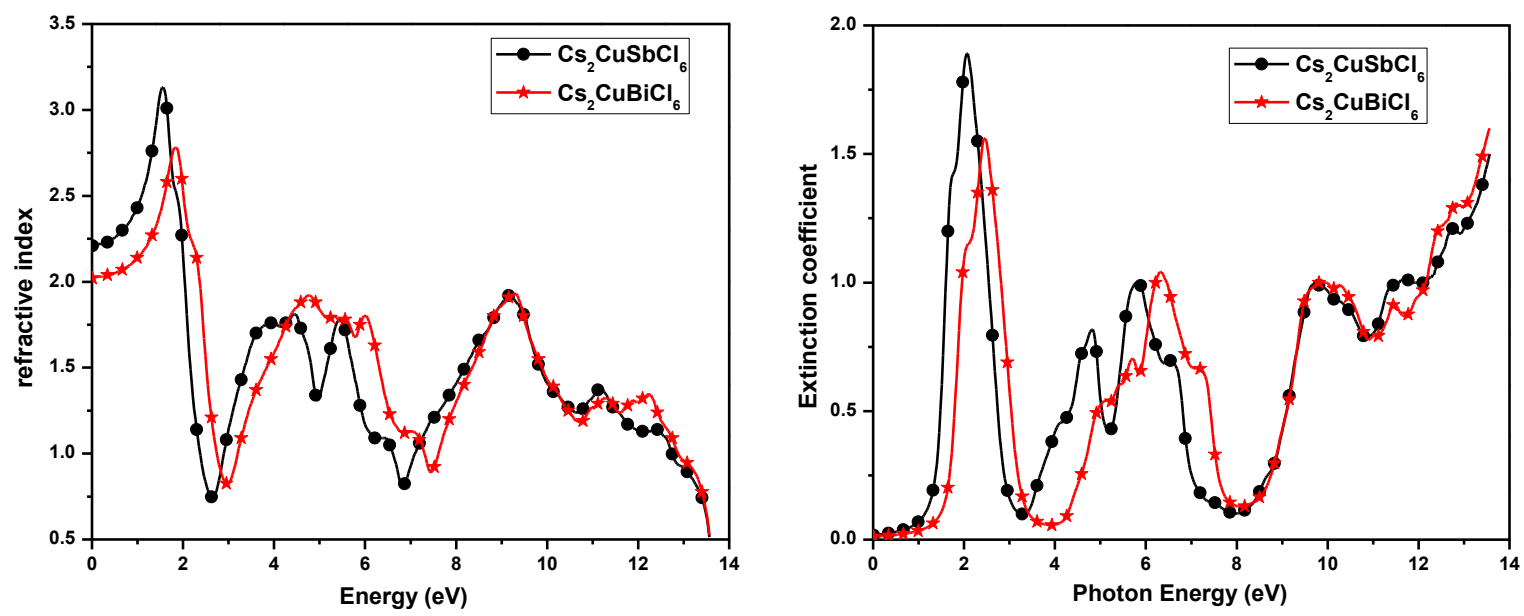

\section{Thermo physical properties:}

The efficiency of thermoelectric materials is directly related by the conversion of thermal energy into the electrical energy and it is characterized by a dimensional less parameter called as figure of merit. The figure of merit is given $Z T=\frac{S^{2} \sigma T}{\kappa_{e l}+\kappa_{l}}$ [59] where $S$ is the Seebeck coefficient, $T$ is temperature, $\sigma$ is electrical conductivity, and $\kappa_{\mathrm{el}}$ and $\kappa l$ are the electron and phonon contributions to total thermal conductivity. To obtain higher thermoelectric efficiency Seebeck coefficient and electrical conductivity must have higher value while as thermal conductivity must possess low value. 
Considering the thermoelectric properties the variation of chemical potential with temperature is interesting. So, to analyze the chemical potential dependence of transport coefficients at different temperatures and hence to characterize various thermoelectric parameters we have used constant relaxation time approximation under BoltzTraP code [34]. First, we started by calculating the variation of carrier concentration with chemical potential at different temperatures depicted in Fig. 4(a). The carrier concentration graph gives us idea about the nature of band structure which describes electronic properties which in turn affects both Seebeck coefficient and thermal conductivity. Since, both these materials show semiconducting nature with no bands at Fermi level giving rise to sharp variation. The sharp variation in carrier concentration corresponds to the presence of band gaps in the band structure. As the temperature increase electron gain more thermal energy hence carrier concentration increases with increase in chemical potential.

As we are already familiar that Seebeck coefficient are extremely sensitive to the value of band gap. The magnitude of Seebeck coefficient is directly related to the dispersion of energy levels near the Fermi level. The Seebeck coefficient (S) as a function of chemical potential $(\square)$ ranging between $-1.5-3 \mathrm{eV}$ at different temperature range $(300 \mathrm{~K}, 600 \mathrm{~K}, 800 \mathrm{~K})$ is plotted in Fig. 4(b). For the entire region of chemical potential the Seebeck coefficient displays prominent peaks and valleys. There are high intensity peaks for the positive potential as well as negative potential at $300 \mathrm{~K}$ and these values decreases at $600 \mathrm{~K}$ and $800 \mathrm{~K}$ because carrier concentration increases and hence Seebeck coefficient decreases with increase in temperature. The most prominent peaks occurs around the $\mu=0$ to $\mu=1$ as the bands are more dispersed resulting in desolation of energy levels around Fermi level and we have less charge carriers around this range. The maximum value of $\mathrm{S}$ is $2000 \mathrm{~V} / \mathrm{K}$ for $\mathrm{Cs}_{2} \mathrm{CuSbCl}_{6}$ and $1500 \mathrm{~V} / \mathrm{K}$ for $\mathrm{Cs}_{2} \mathrm{CuBiCl}_{6}$ at $300 \mathrm{~K}$ respectively. On comparing the results of both these materials we can conclude that $\mathrm{Cs}_{2} \mathrm{CuBiCl}_{6}$ shows better Seebeck coefficient because of presence of larger band gap as Seebeck coefficient varies inversely with carrier concentration. It is observed that $\mathrm{S}$ decreases as temperature increases and shows decent values of Seebeck coefficient for thermoelectric application. Also, we have compared our results with previous results published [37]. There excellent agreement with other theoretical results guides us a way that these materials can be used in thermoelectric applications.

The graphical variation of electronic conductivity $(\sigma)$ as a function of chemical potential at temperature range $300 \mathrm{~K}, 600 \mathrm{~K}$ and $800 \mathrm{~K}$ is demonstrated in Fig. 4(c). Because of absence of 
energy bands around Fermi level reflecting the semiconducting behavior making the area desolate of charge carriers and hence the conductivity vanishes there but below or above the Fermi level the conductivity increases because of energy band smearing. With the change in chemical potential Seebeck and conductivity coefficients shows both increasing as well as decreasing trend. This is because of presence of energy states at some values in band structure but when temperature increases bands smear. When bands smear the effective mass increases thereby increasing the Seebeck coefficient and decreasing conductivity. This phenomenon occurs at highly populated DOS region while as reverse mechanism occurs for low DOS populated region.

The total lattice thermal conductivity comprises of lattice part arising due to lattice vibrations and electronic part arising due to charge carriers. Here, we tried to evaluate both the components of total thermal conductivity with different chemical potentials at a temperature range $300 \mathrm{~K}$, $600 \mathrm{~K}$ and $800 \mathrm{~K}$ as shown in Fig. 4(d). As, electronic thermal conductivity and electronic conductivity both depend on carrier concentration so with change in chemical potential they follows a similar profile. However, the thermal conductivity increases abruptly with temperature compared to electrical conductivity. The results depicted by these agree well with the Wiedemann Franz law which states the proportional relation between them as follows: $k=\sigma L T$ [60].The lattice part of thermal conductivity is calculated with the help of Slack's equation, $\kappa_{l}=\frac{A \theta_{D}^{3} V^{\frac{1}{3}} m}{\gamma^{2} N^{\frac{2}{3}} T}[61]$. The equation suggests that $\kappa_{l}$ is dependent on Debye temperature $\left(\theta_{\mathrm{D}}\right)$, volume $(\mathrm{V})$, average molar mass per atom $(\mathrm{m})$, Grüneisen parameter $(\gamma)$, temperature $(\mathrm{T})$ and number of atoms per unit cell $(\mathrm{N})$. The value of $\mathrm{A}$ is calculated as, $A=\frac{2.43 \times 10^{8}}{1-\frac{0.514}{\gamma}+\frac{0.228}{\gamma^{2}}}$ [62]. The variation of these interdependent quantities such as Grüneisen parameter and Debye temperature with temperature has been plotted in Fig S4(a, b). Debye temperature is an important parameter that characterizes the thermal vibrations in a solid. It is the maximum temperature above which a solid behaves classically and the constituents' exhibit coupled vibrations. The degree or extent of anharmonicity in a crystal is determined by Grüneisen parameter. As the temperature is increased the atoms start vibrating more rigorously which leads 
to an increase in anharmonic effects. From the pots we can see that $\theta_{D}$ decreases while as $\gamma$ increases with increase in temperature. Finally with the help of these interdependent quantities we can evaluate the lattice thermal conductivity from Slack's equation as shown in Fig S4 (c). But we can see that these thermodynamic parameters don't change much with temperature so it is clear that $\mathrm{kl}$ depends mostly on no. of atoms $(\mathrm{N})$.

The most important parameter which scrutinizes the efficiency of thermoelectric materials is the dimensionless figure of merit [63]. The relation of ZT clearly signifies that it increases with electrical conductivity and Seebeck coefficient while it decreases with increasing thermal conductivity. Fig. 4 (e) display the figure of merit (ZT) values of $\mathrm{Cs}_{2} \mathrm{CuSbCl}_{6}$ and $\mathrm{Cs}_{2} \mathrm{CuBiCl}_{6}$ as a function of chemical potential at temperature $300 \mathrm{~K}, 600 \mathrm{~K}$ and $800 \mathrm{~K}$. Both these halide perovskites have prominent peaks with highest peak having ZT nearly equal to 1 . The high value of ZT can be attributed to the semiconducting nature of these materials. We can see from graph that as temperature increases the magnitude of ZT begins to increase and reaches the value of at high temperature.

Fig. 4 (a).Variation of carrier concentration with chemical potential at different temperatures.
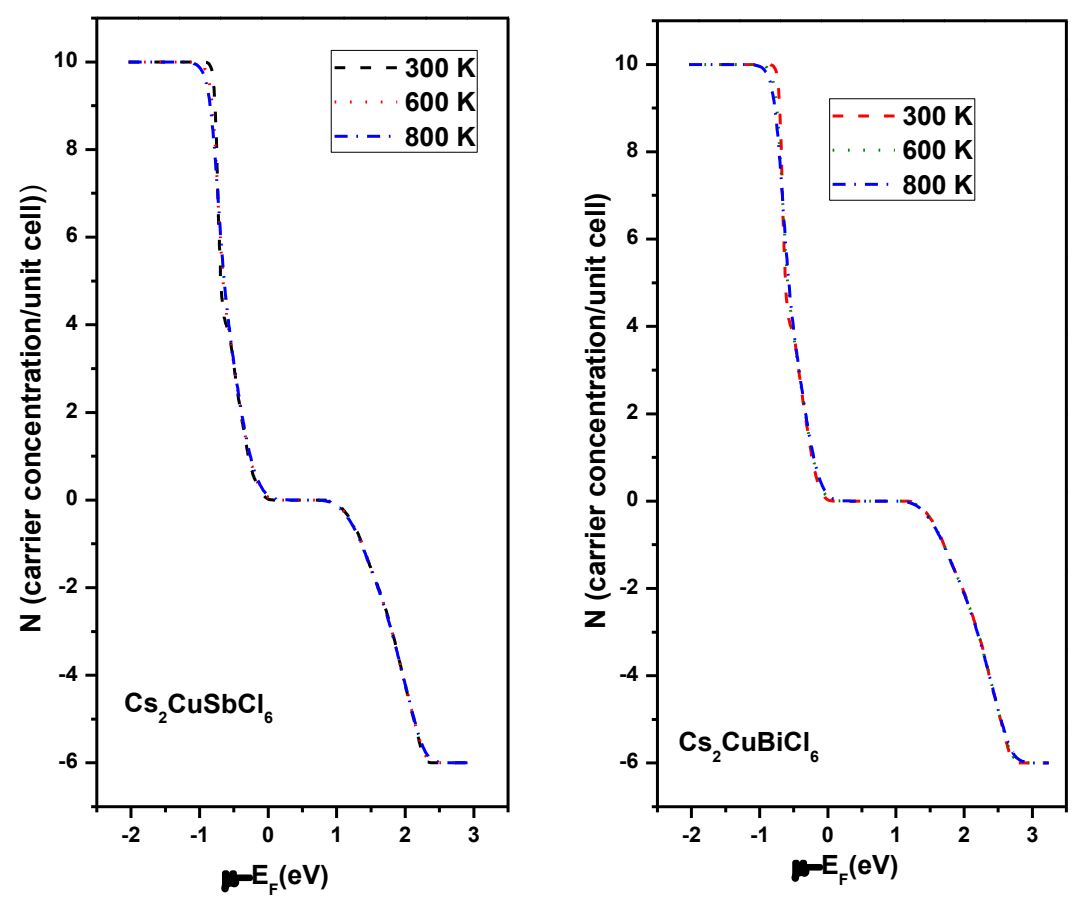

Fig. 4 (b).Variation of Seebeck coefficient with chemical potential at different temperatures. 

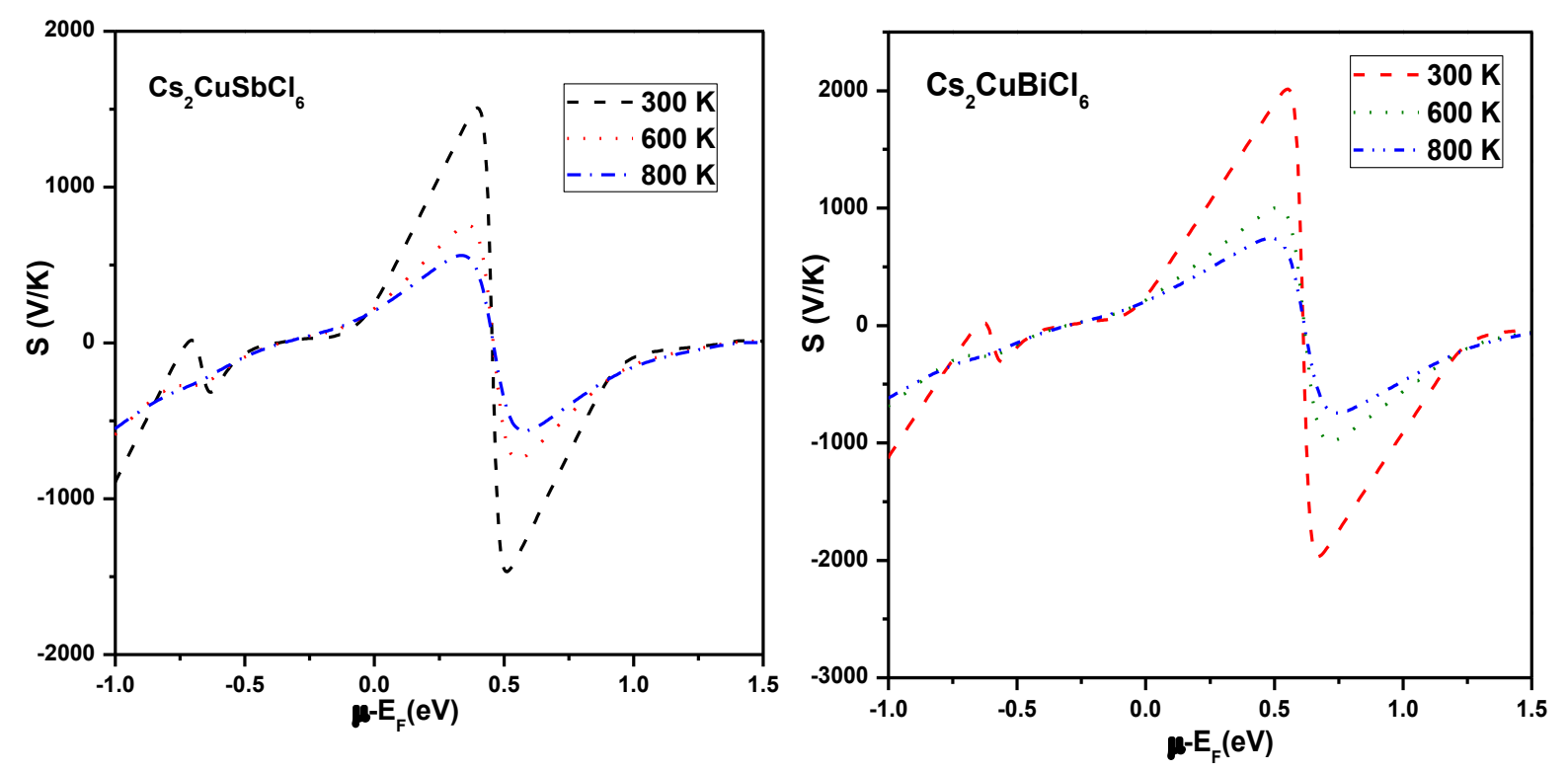

Fig.4(c).Variation of total electronic conduction with chemical potential at different temperatures.
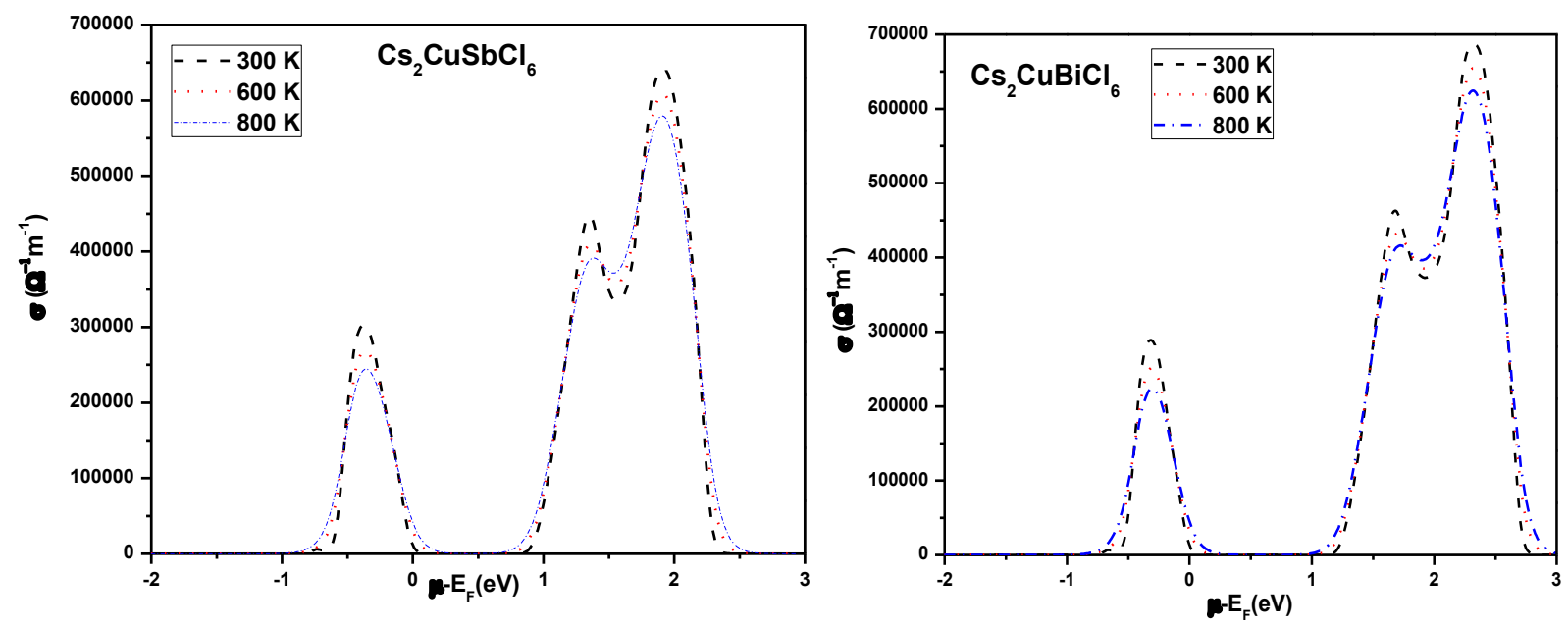

Fig. 4(d).Variation in total electronic thermal conductivity with chemical potential at different temperatures. 

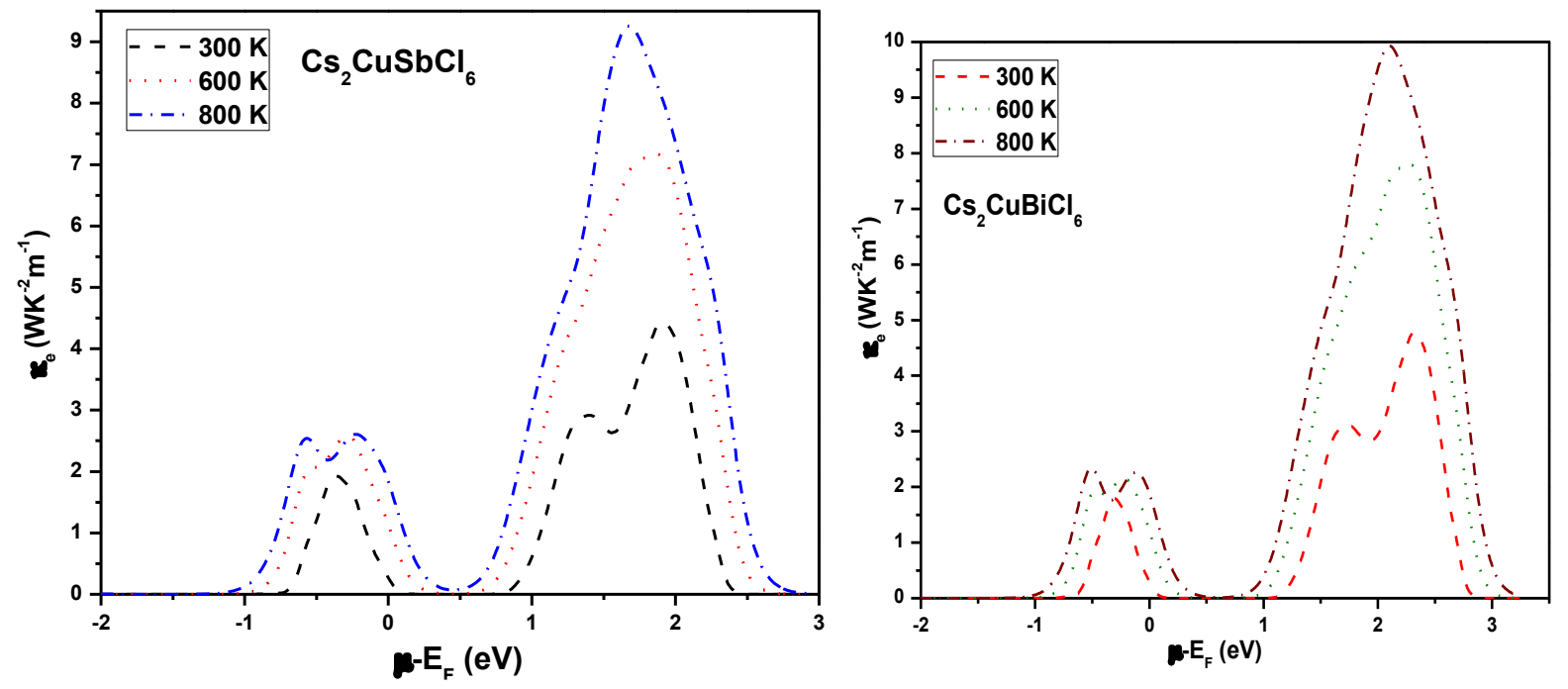

Fig. 4 (e).Variation ZT considering both electronic as well as with chemical potential at different temperatures.
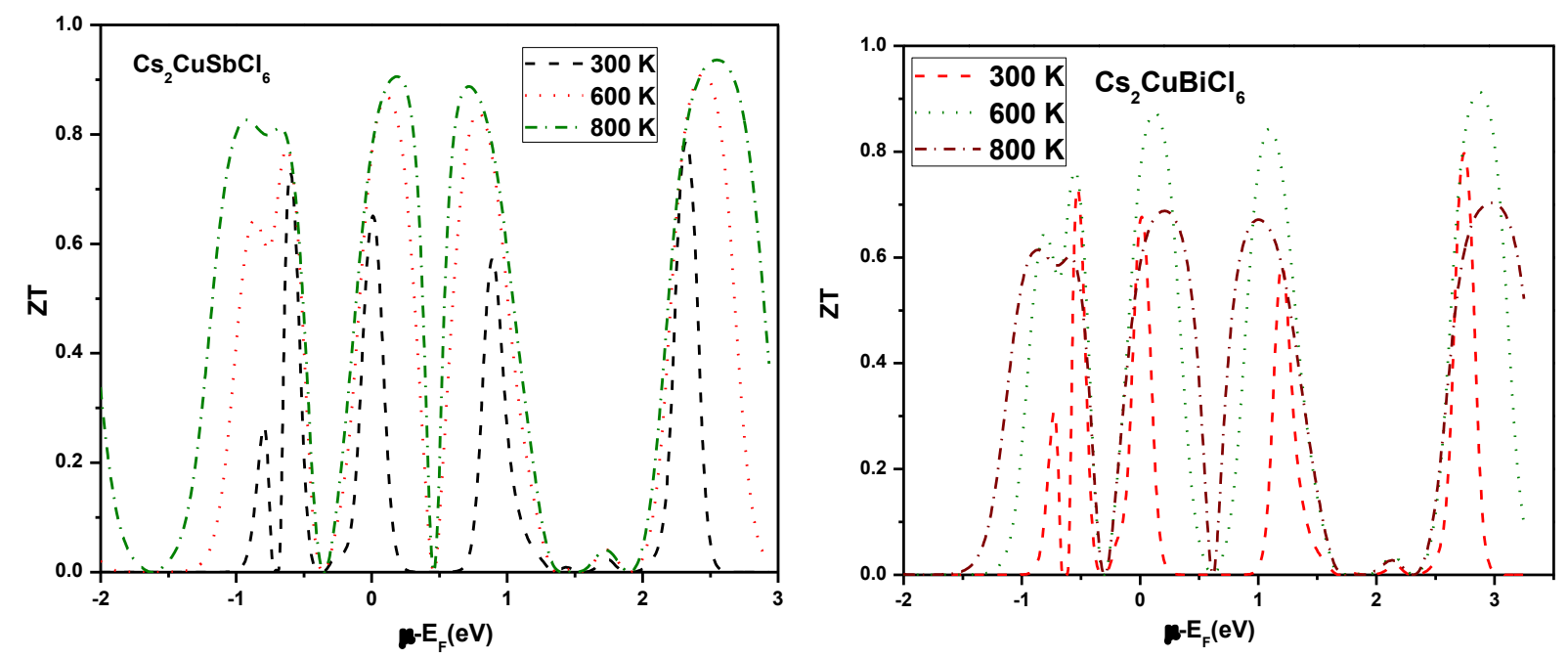

\section{Conclusion}

In the present study the structural stability along with electronic, elastic, thermoelectric and optical properties of inorganic halide double perovskites have been calculated. Both the materials are stable in cubic structure follow the space symmetry of the Fm-3m space group. The stability in the Fm-3m space group is defined with the help of energy optimization, tolerance factor. Moreover, the positive values of elastic constants authenticate the mechanical stability of the materials. The elastic constants further confirm the ductile and anisotropic nature of the materials. The band structure and density of states reflect the semiconducting character with 
small indirect ban gap. These materials have excellent optical absorption in the visible range can be used for solar cell absorbing material. The high Seebeck coefficient with low thermal conductivity is responsible for high figure of merit close to unity.

Conflict of interest: We hereby declare that we don't have any conflict of interest.

Data availability statement: The data would be available from the corresponding author on a reasonable request.

Author contribution statement: Both the authors have significant contributions in conducting this research work. Muskan Nabi has carried out the calculations and wrote the original manuscript. Dr. D. C. Gupta contributed in analysis and discussion for the results. He also helped in improving the current form of manuscript.

\section{References:}

[1] A. Kojima, K. Teshima, Y. Shirai and T. Miyasaka Organometal halide perovskites as visible-light sensitizers for photovoltaic cells. J. Am. Chem. Soc. 131, 6050-6051; (2009).

[2]. Y. Shao, Y. Yuan and J. Huang Correlation of energy disorder and open circuit voltage in hybrid perovskite solar cells. Nat. Energy, 1, 15001; (2016).

[3]. S. S Shin, E. J Yeom, W. S. Yang, S. Hur, M. G. Kim, J. Im, J. Seo, J. H. Noh and S.I. Seok, Colloidally prepared La Doped $\mathrm{BaSnO}_{3}$ electrodes for efficient, photostable perovskite solar cells. Science, 356, 167-171; (2017).

[4]. M. M. Lee, J. Teuscher, T. Miyasaka, T.N. Murakami and H.J. Snaith Efficient hybrid solar cells based on meso super structured organometal halide perovskites Science 338, 643-647; (2012)

[5]. M. Liu, M.B. Johnston and H.J. Snaith Efficient planar heterojunction perovskite solar cells by vapour deposition Nature 501, 395-398; (2013).

[6] F. Igbari, Z. Wang and L. S. Liao Progress of Lead-Free Halide Double Perovskites Adv. Energy Mater. 1803150, 1-32; (2019).

[7] S. E. Creutz, E. N. Crites, M. C. De Siena and D. R. Gamelin, Colloidal nanocrystals of Lead-Free Double-Perovskite (Elpasolite) Semiconductors: Synthesis and anion exchange to access new materials. Nano Letters, 18 (2), 1118-1123; (2018).

[8] E. Meyer, D. Mutukwa , N. Zingwe and R. Taziwa Lead-Free Halide Double Perovskites: A Review of the Structural, Optical, and Stability Properties as Well as Their Viability to Replace Lead Halide Perovskites Metals, 8 (667), 1-16; (2018).

[9] A. Jain, O. Voznyy and E. H. Sargent High-Throughput Screening of Lead-Free Perovskite-like Materials for Optoelectronic Applications J. Phys. Chem. C 121, 7183-7187; (2017).

[10] Z. Zhang, J. Su, J. Hou, Z. Lin, Z. Hu, J. Chang, J. Zhang and Y. Hao Potential Applications of Halide Double Perovskite $\mathrm{Cs}_{2} \mathrm{AgInX}_{6}(\mathrm{X}=\mathrm{Cl}, \mathrm{Br})$ in Flexible Optoelectronics: Unusual Effects of Uniaxial Strains J. Phys. Chem. Lett., 10, 1120-1125; (2019).

[11] S. J. Zelewski, J. M. Urban, A. Surrente, D. K. Maude, A. Kuc, L. Schade, R. D. Johnson, M. Dollmann, P. K. Nayak, H. J. Snaith, P. Radaelli, R. Kudrawiec, R. J. Nicholas, P. Plochocka and M. 
Baranowski Revealing the nature of photoluminescence emission in the metal-halide double perovskite Cs2AgBiBr6 J. Mater. Chem. C, 7, 8350-8356; (2019).

[12] S. Wenwu, T. Cai, Z. Wang and O. Chen The effects of monovalent metal cations on the crystal and electronic structures of $\mathrm{Cs}_{2} \mathrm{MBiCl}_{6}(\mathrm{M}=\mathrm{Ag}, \mathrm{Cu}, \mathrm{Na}, \mathrm{K}, \mathrm{Rb}$, and $\mathrm{Cs})$ perovskites $J$. Chem. Phys. 153, 141101; (2020).

[13] S. Zhao, K. Yamamoto, S. Iikubo, S. Hayase and T. Ma, First-principles study of electronic and optical properties of lead-free double perovskites $\mathrm{Cs}_{2} \mathrm{NaBX}_{6}(\mathrm{~B}=\mathrm{Sb}, \mathrm{Bi} ; \mathrm{X}=\mathrm{Cl}, \mathrm{Br}, \mathrm{I})$. J. Phys. Chem. Solids, 117, 117-121; (2018).

[14] Y. Yin, W. Tian, J. Leng, J. Bian, S. Jin. Carrier Transport Limited by Trap State in $\mathrm{Cs}_{2} \mathrm{AgBiBr}_{6}$ double Perovskites. J. Phys. Chem. Lett. 11 (17), 6956-6963; (2020).

[15] S. Pramchu, A. P. Jaroenjittichai and Y. Laosiritaworn, First-principles investigations of structural stability and electronic band structure of $\mathrm{CH}_{3} \mathrm{NH}_{2} \mathrm{BiI}_{3}$ for lead-free perovskite solar cell application $J$. Phys.: Conf. Ser. 1144, 1-4; (2018).

[16] X.-G Zhao, J.-H Yang, Y. Fu, D. Yang, Q. Xu, L. Yu, S.-H Wei, L. Zhang, Design of Lead-Free Inorganic Halide Perovskites for Solar Cells via Cation-Transmutation. J. Am. Chem. Soc., 139, 26302638; (2017).

[17] W. Meng, X. Wang, Z. Xiao, J. Wang, D. B. Mitzi, Y. Yan, Parity-Forbidden Transitions and Their Impact on the Optical Absorption Properties of Lead-Free Metal Halide Perovskites and Double Perovskites. J. Phys. Chem. Lett. 8, 2999-3007; (2017).

[18] J. Dai, L. Ma, M. Ju, J. Huang, X. Cheng Zeng, In- and Ga based Inorganic Double Perovskites with Direct Band gaps for Photovoltaic Applications. Phys. Chem. Chem. Phys. 19, 21691-21695; (2017).

[19] Y. Cai, W. Xie, Y. T. Teng, P. C. Harikesh, B. Ghosh, P. Huck, K. A. Persson, N. Mathews, S. G. Mhaisalkar, M. Sherburne and M. Asta High-throughput Computational Study of Halide Double Perovskite Inorganic Compounds Chem. Mater, 31, 5392-5401; (2019)

[20] H. Pham, R. Holmes, E. Aydil, L. Gagliardi, Lead-free double perovskites $\mathrm{Cs}_{2} \mathrm{InCuCl}_{6}$ and $(\mathrm{CH} 3 \mathrm{NH} 3)_{2} \mathrm{InCuCl}_{6}$ : electronic, optical, and electrical properties. Nanoscale, 11, 11173-11182; (2019)

[21] G-X. Zhao, D. Yang, Y. Sun, T. Li, L. Zhang, L. Yu, A. Zunger, Cu-In Halide Perovskite Solar Absorbers. J. Am. Chem. Soc., 139, 6718-6725; (2017).

[22] W. Zhou, P. Han, X. Zhang, D. Zheng, S. Yang, Y. Yang, C. Luo, B. Yang, F. Hong, D. Wei, R. Lu and K. Han, Lead-Free Small-Bandgap $\mathrm{Cs}_{2} \mathrm{CuSbCl}_{6}$ Double Perovskite Nanocrystals J. Phys. Chem. Lett., 11; 6463-6467; (2020).

[23] X-G. Zhao, J-H. Yang, Y. Fu, D. Yang, Q. Xu, L.Yu, S-H. Wei and L. Zhang Design of Lead-Free Inorganic Halide Perovskites for Solar Cells via Cation-Transmutation J. Am. Chem. Soc., 139 (7), 2630-2638; (2017).

[24] H-J. Feng, W. Deng, K. Yang, J. Huang, and X. C. Zeng Double Perovskite $\mathrm{Cs}_{2} \mathrm{BBiX} 6(\mathrm{~B}=$ $\mathrm{Ag}, \mathrm{Cu} ; \mathrm{X}=\mathrm{Br}, \mathrm{Cl}) / \mathrm{TiO}_{2}$ Heterojunction: An Efficient Pb-Free Perovskite Interface for Charge Extraction J. Phys. Chem. C, 121, 4471-4480; (2017).

[25]. A. M. Jafar, K. A. Khalaph and A. M. Hmood Lead-free perovskite and double perovskite solar cells Mater .Sci. Eng. 765, 012047; (2020).

[26] X. Che, C. Wang, Z. Li , Z. Hou and W- J. Yin Bayesian optimization based on a unified figure of merit for accelerated materials screening: A case study of halide perovskites Sci China Mater, 63(6), 1024-1035; (2020).

[27] M. Roknuzzaman, C. Zhang, K. Ostrikov, A. Du, H. Wang, L. Wang and T. Tesfamichael Electronic and optical properties of lead-free hybrid double perovskites for photovoltaic and optoelectronic applications. Sci. Rep., 9, 718; (2019)

[28] M. Roknuzzaman, J. A. Alarco, H. Wang, K. Ostrikov Structural, electronic and optical properties of lead-free antimony-copper based hybrid double perovskites for photovoltaics and optoelectronics by first principles calculations Comput. Mater. Sci. 186, 1100092; (2021). 
[29] E. Meyer, D. Mutukwa , N. Zingwe and R. Taziwa Lead-Free Halide Double Perovskites: A Review of the Structural, Optical, and Stability Properties as Well as Their Viability to Replace Lead Halide Perovskites Metals 8, 667; (2018).

[30] G. Volonakis, M. R. Filip, A. A. Haghighirad, N. Sakai, B. Wenger, H. J Snaith, F. Giustino Lead-Free Halide Double Perovskites via Heterovalent Substitution of Noble Metals J.Phys. Chem Lett 7(7), 1254-1259; (2016).

[31] K. Schwarz, P. Blaha and G. K. H. Madsen, Electronic structure calculations of solids using the WIEN2k package for material sciences. Comput. Phys. Commun., 147(1-2), 71-76; (2002).

[32] P. J. Perdew, K. Burke and M. Ernzerhof, Generalized Gradient Approximation Made Simple. Phys. Rev. Lett 77 (18), 3865-3868; (1996).

[33] T. Rezkallah, I. Djabri, M. M. Koç, M. Erkovan, Y. Chumakov, F. Chemam Investigation of the electronic and magnetic properties of Mn doped $\mathrm{ZnO}$ using the FP-LAPW method Chin. J. Phys 55 (4), 1432-1440; (2017).

[34] G. K. H Madsen, and D. J. Singh, BoltzTraP. A code for calculating band-structure dependent quantities Comput Phys Commun., 175 (1), 67-71; (2006).

[35] A.O-D-L-Roza, D. A.-Pérez, V. Luaña GiBBs2: A new version of the quasi-harmonic model code. II. Models for solid-state thermodynamics, features and implementation Comput. Phys. Commun 182, 2232-2248; (2011).

[36] T. Katsura and Y. Tange A Simple Derivation of the Birch-Murnaghan Equations of State (EOSs) and Comparison with EOSs Derived from Other Definitions of Finite Strain Minerals 9(12), 745; (2019).

[37] S. A. Mir and D. C. Gupta Analysis of cage structured halide double perovskites $\mathrm{Cs}_{2} \mathrm{NaMCl}_{6}(\mathrm{M}=\mathrm{Ti}, \mathrm{V})$ by spin polarized calculations J Alloys Compd., 854, 1-10; (2020).

[38] S. F. Hoefler, G. Trimmel and T. Rath Progress on lead-free metal halide perovskites for photovoltaic applications: a review Monatsh Chem 148, 795-826; (2017).

[39] M.E. Fine, L.D. Brown, H.L. Marcus, Scr. Metall. 18 (1984) 951.

[40] I. Khan, I. Ahmad, R.A.H Aliabad and M. Maqbool,. DFT-mBJ Studies of the Band

Structures of the II-VI Semiconductors. Materials Today: Proceedings, 2(10), (2015) 5122-

5127.

[41] W.Chatta, B. Lagoun, H., Lidjici, A.Chadli, A.Cheriet, H.Farh and K.Salah, TB-mBJ Calculations of Structural and Optoelectronic Properties of the Rhombohedral Phase of Bismuth Sodium Titanate (Bi0.5 Na0.5)TiO3. Solid State Phenom. 297, (2019). 165-172.

[42] M. Jamal, Cubic-elastic, http://www.WIEN2k.at/reg_user/unsupported/cubic-elast/ (2012).

[43] M. Nabi, D. C. Gupta Small-band gap halide double perovskite for optoelectronic properties Int J Energy Res. (2020); 1-13 doi:10.1002/er.6307.

[44] M. Born, On the stability of crystal lattices. I. Math. Proc. Cambridge Philos. Soc., (1940), 36, 160, DOI: $10.1017 /$ s0305004100017138.

[45] I. S. Ranganathan, M.O-Starzewski, Universal elastic anisotropy index. Phys. Rev. Lett. (2008), 101, 055504.

[46] X. Luan, H. Qin, F. Liu, Z. Dai, Y. Yi and Q. Li The Mechanical Properties and Elastic Anisotropies of Cubic Ni3Al from First Principles Calculations Crystals (2018), 8, 307.

[47] R. Gaillac, P. Pullumbi and F-X. Coudert, ELATE: an open source online application for analysis and visualization of elastic tensors, J. Phys.: Condens. Matter, 28, 275201;(2016) DOI: 10.1088/0953-8984/28/27/275201.

[48] R. Hill, The elastic behaviour of a crystalline aggregate. Proc. Phys. Soc. 65, 394-354; (2002) 
[49] S. F. Pugh, XCII. Relations between the elastic moduli and the plastic properties of polycrystalline pure metals. Philos. Mag. (2009), 45, 823-843.

[50] B. Huang, Y. H. Duan, W.C. Hu, Y. Sun, S. Chen, Structural, anisotropic elastic and thermal properties of $\mathrm{Mb}(\mathrm{M}=\mathrm{Ti}, \mathrm{Zr}$ and $\mathrm{Hf}$ ) monoborides. Ceram. Int. (2015), 41, 6831-6843.

[51] M. J. Zhang, Y. Zhang, W. K. Xu, V. Ji Young's modulus surface and poisson's ratio curve forcubic metals. J. Phys. Chem. Solids (2007), 68, 503-510.

[52] T. M. Bhat and D. C. Gupta, Transport, Structural and Mechanical Properties of Quaternary FeVTiAl Alloy. J. Electron. Mater. (2016). 45(11), 6012-6018.

[53] S. A. Khandy and D. C. Gupta Study of ferromagnetism, spin-polarization, thermoelectrics and thermodynamics of layered perovskite $\mathrm{Ba}_{2} \mathrm{FeMnO}_{6}$ under pressure and temperature Phys. Status Solidi B (2019), 1800625 1-7

[54] O.L. Anderson, J. Phys. Chem. Solids 24 (1963) 909.

[55] P. Ravindran, L. Fast, P.A. Korzhavyi, B. Johansson, J. Wills, O. Eriksson, J. Appl. Phys. 84 (1998) 4891.

[56] U-G. Jong, C-J. Yu and Y-H. Kye Computational prediction of structural, electronic, and optical properties and phase stability of double perovskites $\mathrm{K}_{2} \mathrm{SnX}_{6}(\mathrm{X}=\mathrm{I}, \mathrm{Br}, \mathrm{Cl}) R S C$ Adv., $\mathbf{1 0}$, 201-209; (2020).

[57] M. Fox, Optical properties of solids. Am. J. Phys. 70, 1269; (2002)

[58] P. Jiang, P. Boulet and M-C. Record A DFT study of the electronic, optical and topological properties of free and biaxially strained $\mathrm{CuIn}_{1-x} \mathrm{Al}_{x} \mathrm{Se}_{2}$ J. Mater. Chem. C, 7, 5803-5815; (2019),

[59] T. M. Bhat and D. C. Gupta Magneto-electronic, thermal, and thermoelectric properties of some Co-based quaternary alloys J. Phys. Chem. Solids 112, 190-199; (2018).

[60] W. Kim Strategies for engineering phonon transport in thermoelectrics J. Mater. Chem. C, 3, 10336-10348; (2015).

[61] P. B. Allen, Zero-point and isotope shifts: Relation to thermal shifts, Phil. Mag. B 70 (3), 527- 534; (1994).

[62] P. Nath, J. J. Plata, D. Usanmaz, C. Toher, M. Fornari, M. B. Nardelli and S. Curtarolo, High throughput combinatorial method for fast and robust prediction of lattice thermal conductivity. Scr. Mater., 129, 88-93; (2017).

[63] S.A. Khandy and D. C. Gupta (2019). Magneto-electronic, mechanical, thermoelectric and thermodynamic Properties of Ductile Perovskite $\mathrm{Ba}_{2} \mathrm{SmNbO}_{6}$. Mater. Chem. Phys.239, 121983; (2020). 
Figures
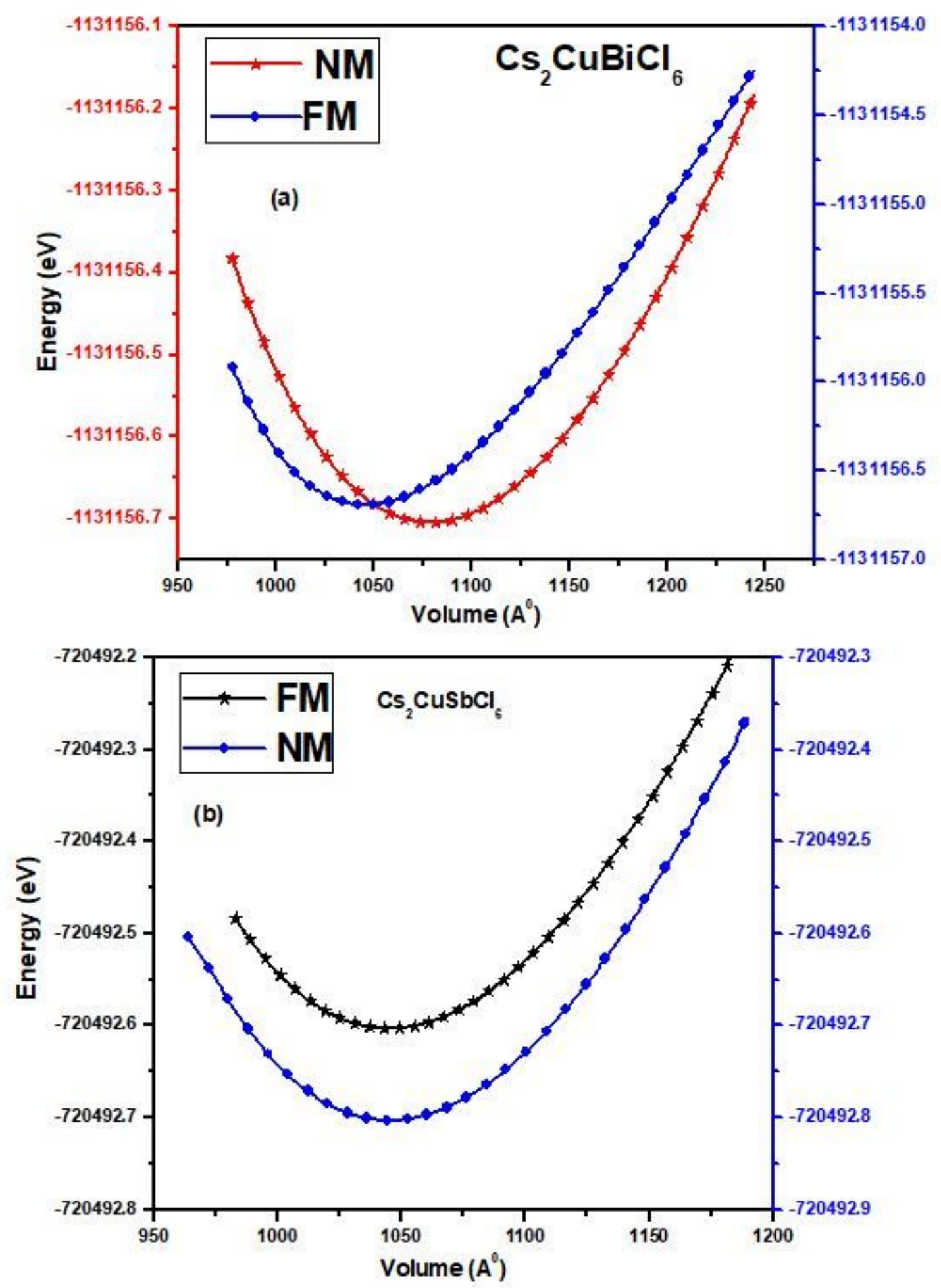

Figure 1

$(\mathrm{a}, \mathrm{b})$ : Energy versus volume optimization curve of $\mathrm{Cs} 2 \mathrm{CuMCl} 6(\mathrm{M}=\mathrm{Sb}, \mathrm{Bi})$ in both spin polarized and nonpolarized states. 

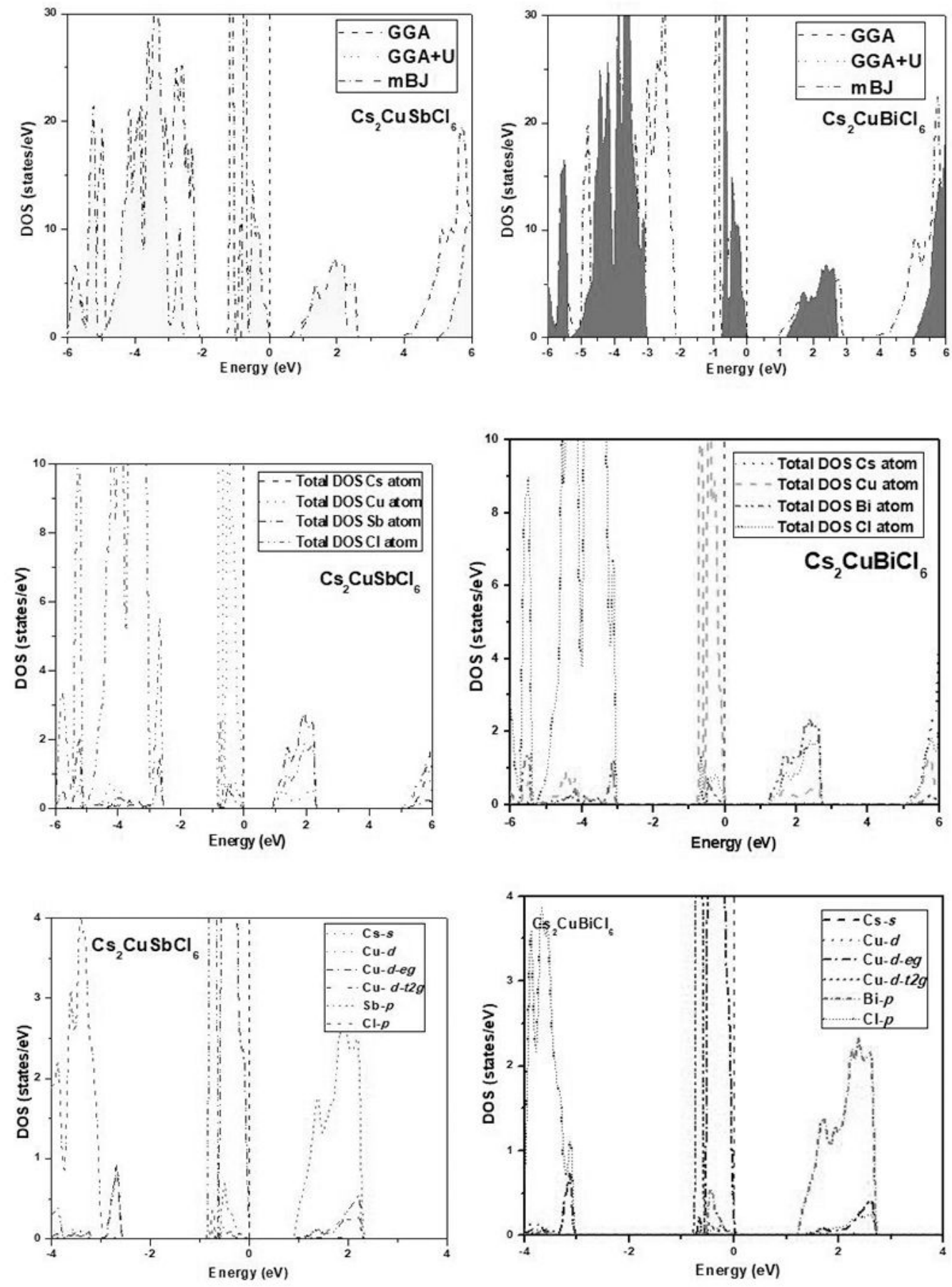

Figure 2

(a): Density of states for Cs2CuMCl6 ( $\mathrm{M}=\mathrm{Sb}, \mathrm{Bi})$ calculated by GGA, GGA+U and mBJ scheme. (b): Partial density of states pDOS for Cs2CuMCl6 (M=Sb, Bi) calculated by mBJ scheme. (c): Partial density of states pDOS for Cs2CuMCl6 ( $\mathrm{M}=\mathrm{Sb}, \mathrm{Bi})$ calculated by mBJ scheme. 

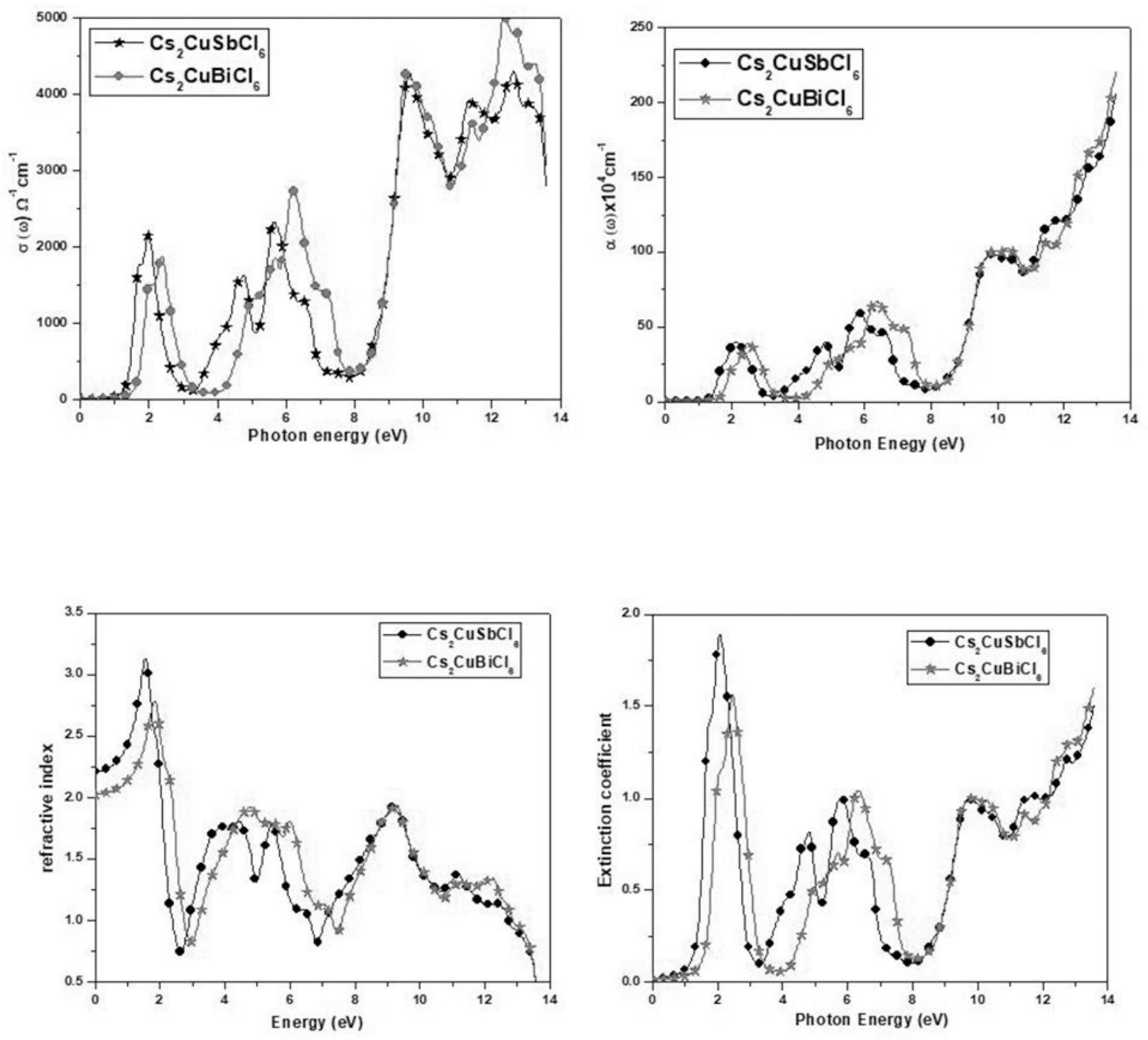

\section{Figure 3}

$(a, b)$ : variation of optical absorption and conductivity with photon Energy. $(c, d)$ : variation of refractive index and extinction coefficient with photon Energy. 

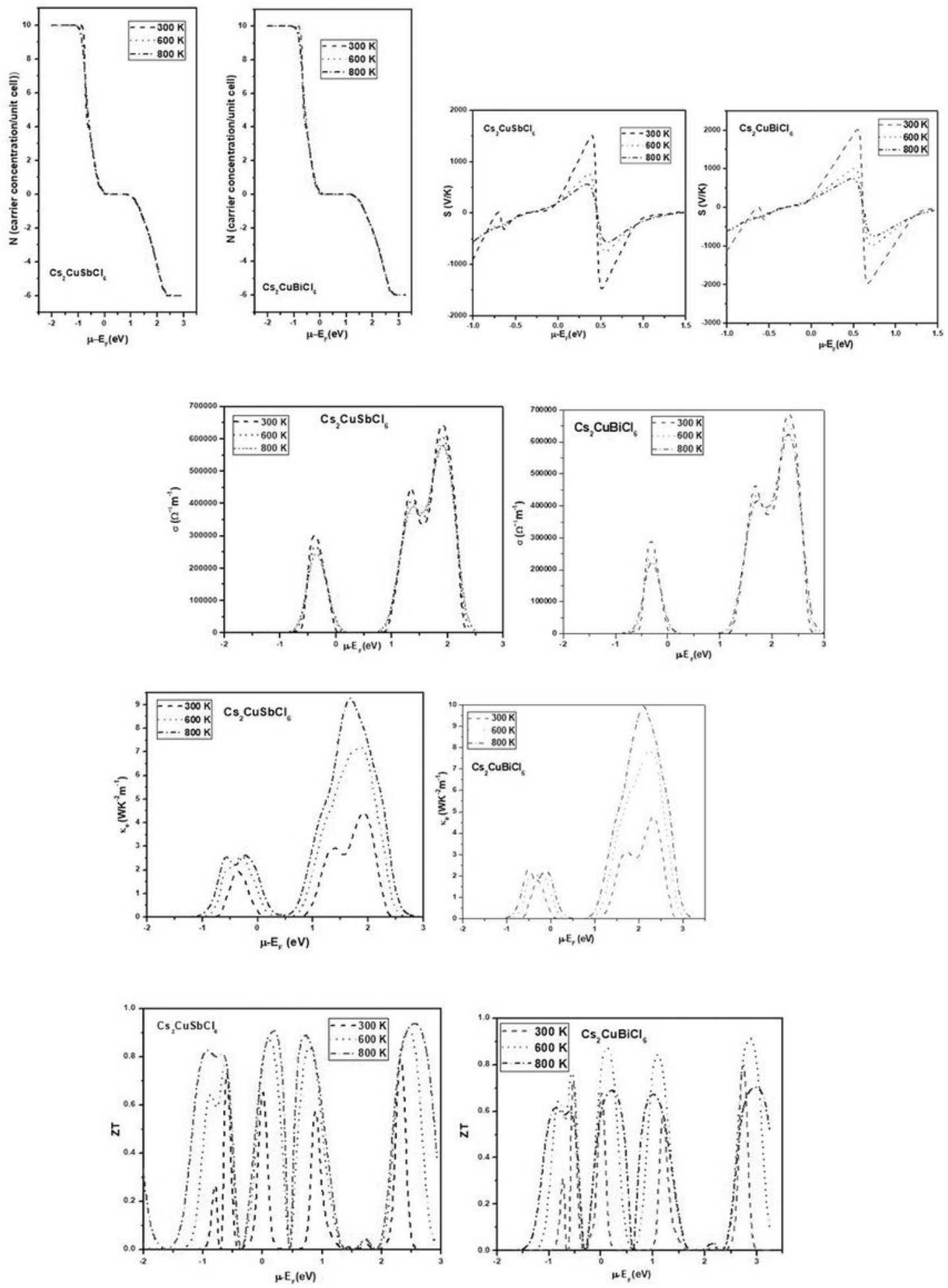

\section{Figure 4}

(a).Variation of carrier concentration with chemical potential at different temperatures. (b).Variation of Seebeck coefficient with chemical potential at different temperatures. (c).Variation of total electronic conduction with chemical potential at different temperatures. (d).Variation in total electronic thermal conductivity with chemical potential at different temperatures. (e).Variation ZT considering both electronic as well as with chemical potential at different temperatures. 


\section{Supplementary Files}

This is a list of supplementary files associated with this preprint. Click to download.

- SupplementaryinformationCs2CuMCl6.docx 